\title{
Macrophage alterations in islets of obese mice linked to beta cell disruption in diabetes
}

\author{
Jeng Yie Chan ${ }^{1} \cdot$ Kailun Lee $^{1} \cdot$ Emma L. Maxwell ${ }^{1} \cdot$ Cassandra Liang $^{1} \cdot$ D. Ross Laybutt ${ }^{1}$
}

Received: 2 November 2018 / Accepted: 12 February 2019 / Published online: 4 March 2019

(C) Springer-Verlag GmbH Germany, part of Springer Nature 2019

\begin{abstract}
Aims/hypothesis Mild islet inflammation has been suggested as a contributing factor to beta cell failure in type 2 diabetes. Macrophage levels are elevated in the islets of humans and mice with type 2 diabetes, but their effects on beta cells are not understood. Our goal was to examine the gene expression changes in islet-associated macrophages in obesity models with opposing disposition to diabetes development and to assess their potential contribution to beta cell (mal)adaptation.

Methods Islets were isolated from lean control mice, obese diabetes-prone $d b / d b$ mice and obese diabetes-resistant $o b / o b$ mice. Macrophages were sorted using flow cytometry. Islets were treated ex vivo with clodronate-containing liposomes to deplete macrophages. Gene expression was assessed by real-time RT-PCR.

Results Macrophage levels were increased in islets from $d b / d b$ mice but not in islets from $o b / o b$ mice compared with lean control mice. Macrophages from $d b / d b$ and $o b / o b$ islets displayed distinct changes in gene expression compared with control islet macrophages, suggesting differential shifts in functional state. Macrophages from $d b / d b$ islets displayed increased expression of interferon regulatory factor 5 (Irf5), IL-1 receptor antagonist (Ill rn) and mannose receptor C-type 1 (Mrc1), whereas macrophages from $o b / o b$ islets showed elevated levels of transforming growth factor beta 1 (Tgfbl) and reduced IL-1 $\beta$ (Illb). Clodronate-liposome treatment of islets depleted macrophages, as evidenced by reduced mRNA expression of Cd11b (also known as Itgam) and F4/80 (also known as Adgre1) compared with PBS-liposome-treated islets. The depletion of macrophages in $d b / d b$ islets increased the expression of genes related to beta cell identity. The mRNA levels of islet-associated transcription factors (Mafa and $P d x 1$ ), glucose transporter (Glut2 [also known as Slc2a2]), ATP-sensitive $\mathrm{K}^{+}$channel (Kcnj11), incretin receptor (Gipr) and adaptive unfolded protein response (UPR) genes (Xbpl, Hspa5, Pdia4 and Fkbpl1) were increased in db/ $d b$ islets after macrophage depletion, whereas the mRNA levels of the deleterious UPR effector, Ddit 3 , were reduced. In contrast, depletion of macrophages in islets of $o b / o b$ mice did not affect beta cell identity gene expression.

Conclusions/interpretation The findings of this study suggest that distinct alterations in islet macrophages of obese mice are critically important for the disruption of beta cell gene expression in diabetes.
\end{abstract}

Keywords Beta cell $\cdot$ Gene expression $\cdot$ Inflammation $\cdot$ Islets $\cdot$ Macrophage $\cdot$ Type 2 diabetes $\cdot$ Unfolded protein response

\section{Abbreviation \\ UPR Unfolded protein response}

Electronic supplementary material The online version of this article (https://doi.org/10.1007/s00125-019-4844-y) contains peer-reviewed but unedited supplementary material, which is available to authorised users.

D. Ross Laybutt r.laybutt@garvan.org.au

1 Garvan Institute of Medical Research, St Vincent's Clinical School, UNSW Sydney, 384 Victoria St, Darlinghurst, Sydney, NSW 2010, Australia

\section{Introduction}

Pancreatic beta cell compensation for obesity-associated insulin resistance is crucial for the maintenance of glucose homeostasis. Type 2 diabetes develops only in individuals who are unable to sustain the beta cell compensatory response. Beta cell failure in humans and rodents with type 2 diabetes is characterised by the disruption of beta cell function and gene expression $[1,2]$. The latter features the downregulation of beta cell enriched genes, including key transcription factors, beta cell function genes and adaptive unfolded protein 


\section{Research in context}

\section{What is already known about this subject?}

- Islet macrophage levels are elevated in type 2 diabetes

- Islets in type 2 diabetes have reduced expression of beta cell identity and unfolded protein response (UPR) genes

\section{What is the key question?}

- What are the characteristics of islet-associated macrophages in obesity models with opposing disposition to diabetes development (diabetes-prone $d b / d b$ and diabetes-resistant $o b / o b$ mice) and what is their potential influence on beta cell (mal)adaptation?

\section{What are the new findings?}

- Islet macrophages from diabetes-prone and diabetes-resistant obese mice display distinct changes in gene expression compared with control islet macrophages, suggesting differential shifts in functional state

- Macrophage depletion in islets from diabetes-prone $d b / d b$ mice partially restores the changes in beta cell identity and UPR genes

- Macrophage depletion in islets of diabetes-resistant ob/ob mice does not affect islet gene expression

\section{How might this impact on clinical practice in the foreseeable future?}

- These novel findings suggest that distinct alterations in islet macrophages in obesity accompany beta cell compensation and failure. Depleting islet macrophages could be beneficial for restoring beta cell identity in type 2 diabetes

response (UPR) genes [1-4]. However, the triggering stimuli are poorly understood.

Inflammatory stress has been suggested to play a role in the pathogenesis of beta cell failure and type 2 diabetes [5-7]. The number of islet macrophages is increased in type 2 diabetes and they have been proposed as key contributors to beta cell inflammation [5-7]. However, the current knowledge of the molecular differences between islet macrophages in conditions of beta cell compensation and failure is poor. Here we used well-established obese mouse models with opposing disposition to diabetes development (diabetes-resistant $\mathrm{ob} / \mathrm{ob}$ mice and diabetes-prone $d b / d b$ mice) [4] to examine the gene expression changes in flow-sorted islet macrophages and the potential involvement of islet macrophages in beta cell (mal)adaptation.

\section{Methods}

Mice Sixteen-week-old male and female C57BL/KsJ $d b / d b$ and $\mathrm{C} 57 \mathrm{BL} / 6 \mathrm{~J} o b / o b$ mice, and their littermate lean controls (C57BL/KsJ or C57BL/6J, respectively) were taken from the Garvan Institute breeding colonies (Australian BioResources, Moss Vale, NSW, Australia). Animals were kept under conventional conditions with free access to food and water. Procedures were approved by the Garvan Institute/St Vincent's Hospital Animal Experimentation Ethics Committee, following guidelines issued by the National
Health and Medical Research Council of Australia. Islets were isolated by liberase digestion (Roche Diagnostics, Castle Hill, Australia), gradient centrifugation (Ficoll-Paque PLUS gradient; GE Healthcare Bio-Sciences, Uppsala, Sweden) and handpicking under a stereomicroscope [4].

Flow cytometry and cell sorting Flow cytometry and cell sorting of macrophages were performed in a BD FACSAria III cell sorter (Becton Dickinson, Franklin Lakes, NJ, USA) for cells stained positive with F4/80-APC (1:250 dilution;

Fig. 1 Changes in macrophage content and gene expression levels in islets from male and female $d b / d b$ and $o b / o b$ mice. Isolated islets were dissociated into single cell suspensions using trypsin, blocked, and then stained for macrophage-specific markers using F4/80-APC and CD11bFITC antibodies. (a-d) Subsequently, cells were sorted using a BD FACSAria III cell sorter. An example of the flow cytometry gating is shown. Four levels of gating (labelled 'Cells' and 'P2'-'P4') were used to separate single cells from the cell suspension (a). Live cells were then gated from the single cell population with DAPI staining (b). From live single cells, macrophages were gated on F4/80 and CD11b (c). A summary of the results of the gating shown in $(\mathbf{a}-\mathbf{c})$ is given in the table (d). The percentage of gated cells from the parent population is given under the column heading '\%Parent'. Macrophage content was expressed as a percentage of total live islet cells (e). Total RNA was extracted from macrophages, reverse-transcribed and analysed by realtime RT-PCR. Shown are mRNA levels in macrophages of $d b / d b$ (white bars) and $o b / o b$ (grey bars) mouse islets expressed as fold change of the levels in respective controls (represented by the dashed line) (f). All results are mean \pm SEM. $* p<0.05, * * p<0.01, * * * p<0.001$ vs control. Mac, macrophages 
a
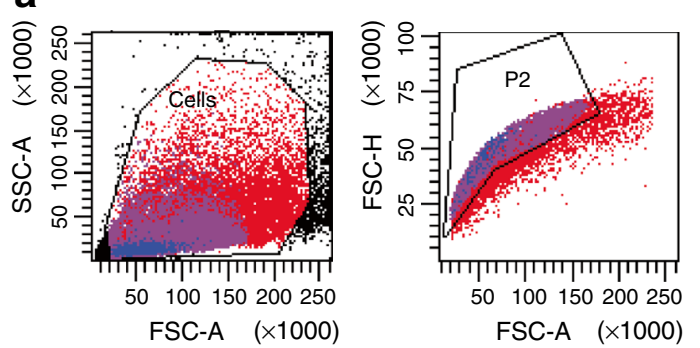

b

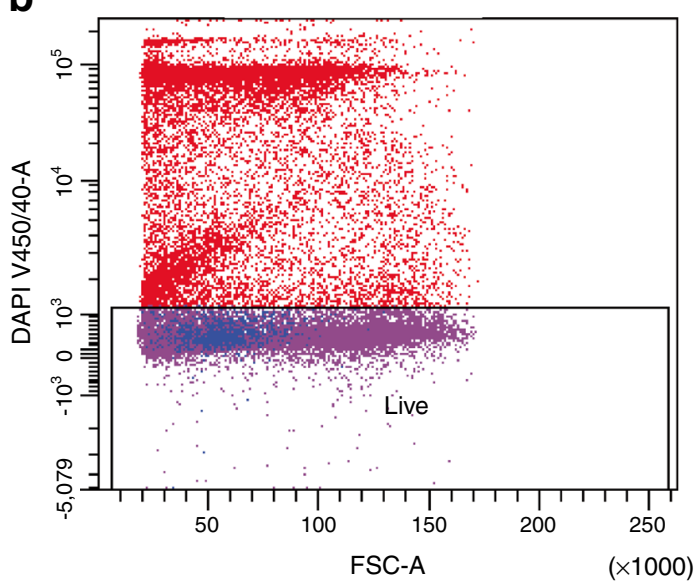

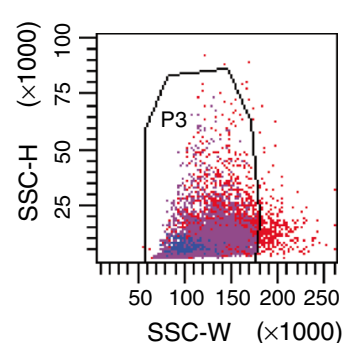

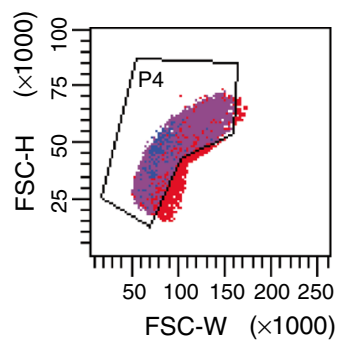

C

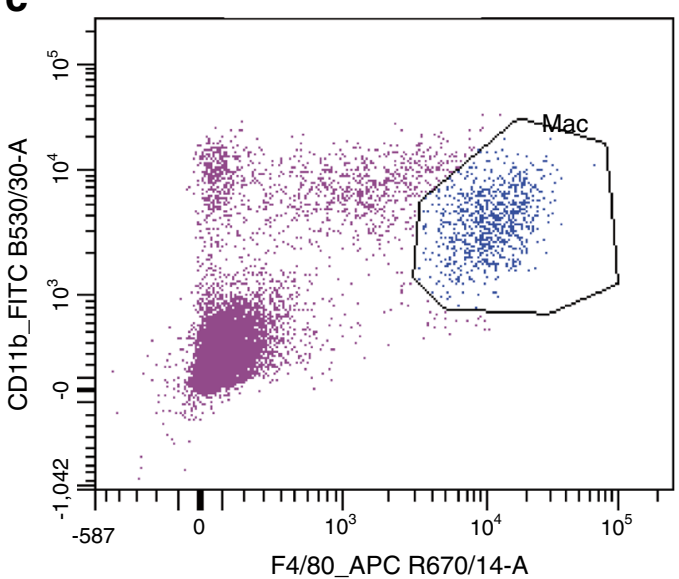

d

\begin{tabular}{|c|c|c|c|}
\hline $\begin{array}{l}\text { Tube: db1 } \\
\text { Population }\end{array}$ & $\begin{array}{l}\text { No. of } \\
\text { events }\end{array}$ & \%Parent & $\%$ Total \\
\hline All events & 89,700 & -- & 100.0 \\
\hline :.... Cells & 53,311 & 59.4 & 59.4 \\
\hline$\square \mathrm{P} 2$ & 39,519 & 74.1 & 44.1 \\
\hline$\square$ P3 & 37,570 & 95.1 & 41.9 \\
\hline$\square \square$ P4 & 31,262 & 83.2 & 34.9 \\
\hline$\square$ Live & 15,226 & 48.7 & 17.0 \\
\hline Mac & 859 & 5.6 & 1.0 \\
\hline
\end{tabular}
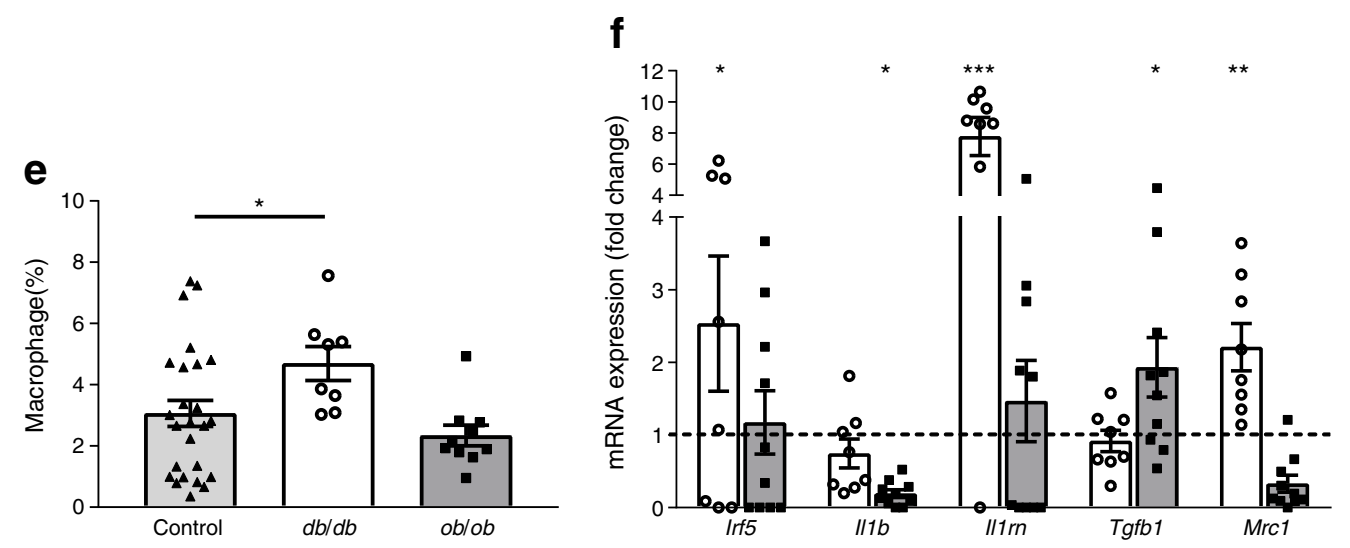

catalogue no. 123115) and CD11b-FITC (1:200 dilution; catalogue no. 101205) antibodies (Biolegend, San Diego, CA, USA) (see Fig. 1a-d and electronic supplementary material [ESM] Methods for further details). DAPI $(0.1 \mu \mathrm{g} / \mathrm{ml})$ was added to samples prior to sorting. Initial gating was applied to capture all cells, followed by single-cell gating. DAPInegative cells were gated as live cells followed by macrophage gating with positive CD11b and F4/80 staining. All 

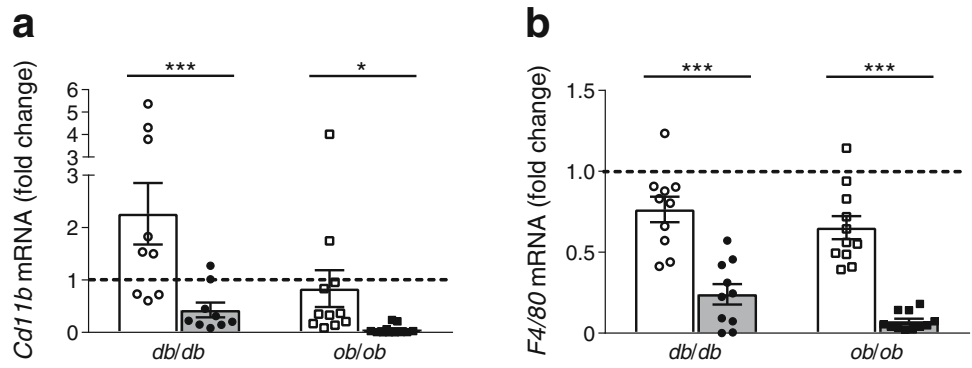

C

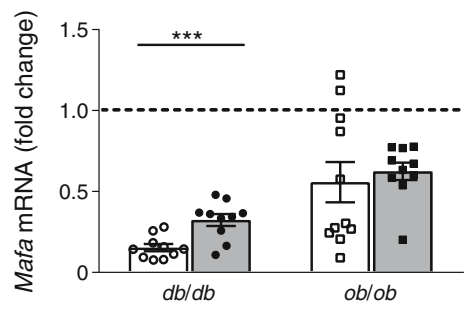

d

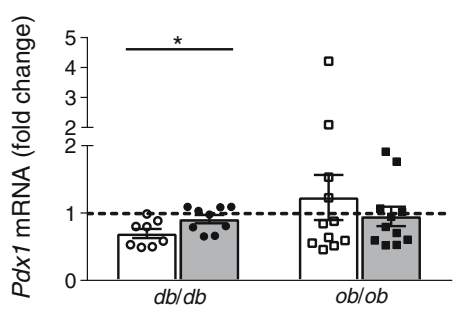

g
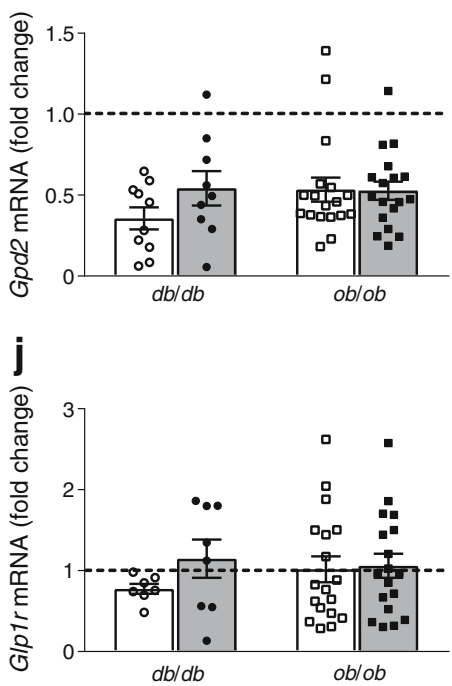

m

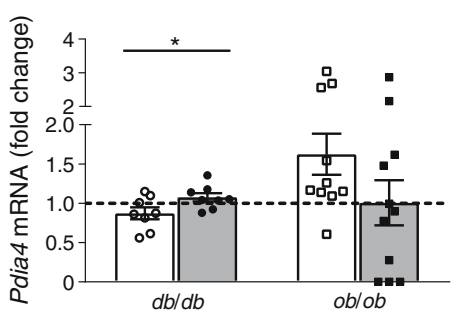

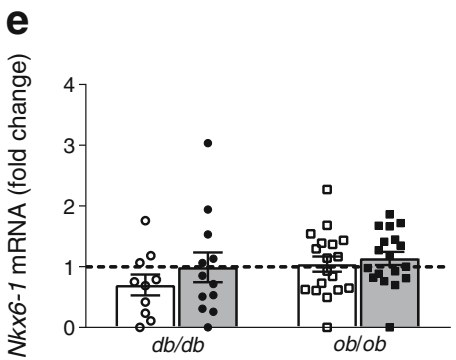

h
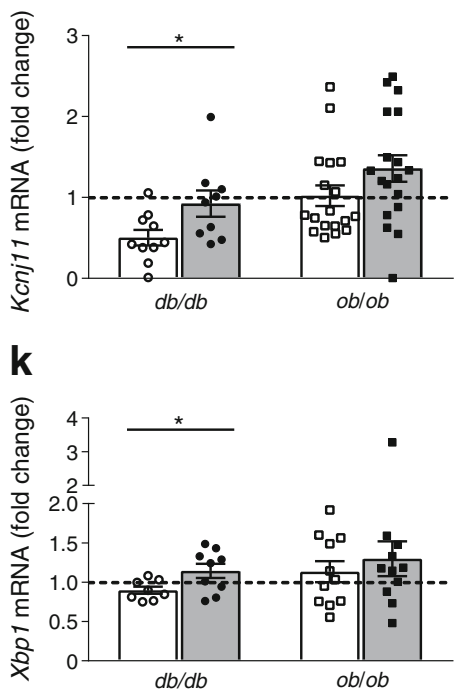

n

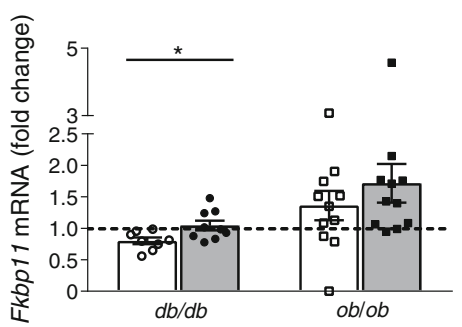

f

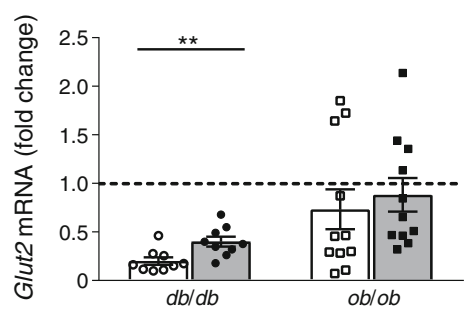

i
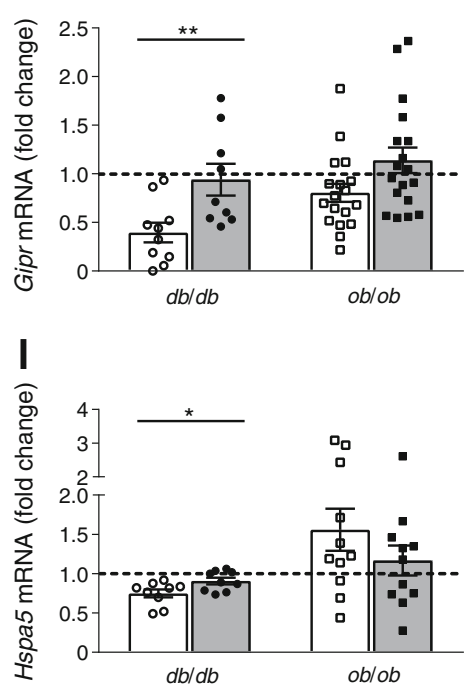

0

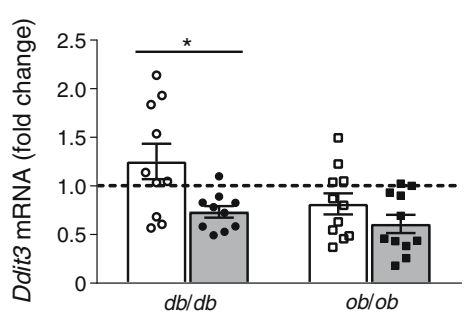

Fig. 2 Effects of macrophage depletion on gene expression in islets from male and female $d b / d b$ and $o b / o b$ mice. Isolated islets were cultured with liposomes containing PBS or clodronate for 2 days. Total RNA was extracted from islets, reverse-transcribed and analysed by real-time RTPCR. Shown are mRNA levels in $d b / d b$ and $o b / o b$ mouse islets cultured in the presence of PBS-liposome (white bars) or clodronate-liposome (grey bars) expressed as fold change of the levels in respective controls (represented by the dashed line). All results are mean \pm SEM. ${ }^{*} p<0.05$, $* * p<0.01, * * * p<0.001$, clodronate-liposome treatment vs PBS-liposome treatment per group gating boundaries were determined using unstained samples. Where possible, sorted cells were re-analysed to ensure collected samples contained only cells that were stained positive for both markers. 
Macrophage depletion in islets Islets were cultured in liposomes containing either PBS or clodronate $(1 \mathrm{mg} / \mathrm{ml})$ (Liposoma, Amsterdam, the Netherlands) for 2 days. Islets were randomly handpicked for treatment. RNA was extracted for gene expression analysis. Insulin and proinsulin secretion was assessed in groups of five islets, as previously described [4]. Insulin was measured by a homogeneous time-resolved fluorescence (HTRF) assay kit (Cisbio; Genesearch, Arundel, QLD, Australia). Proinsulin was measured by ELISA (Mercodia AB, Uppsala, Sweden).

RNA analysis Total RNA from macrophages and islets was extracted using the RNeasy Micro Kit and RNeasy Mini Kit, respectively (Qiagen, Doncaster, VIC, Australia). cDNA was synthesised using the QuantiTect Reverse Transcription Kit (Qiagen). Real-time PCR with macrophage and islet cDNA was performed on a 7900HT Real-Time PCR System (Applied Biosystems, Foster City, CA, USA) using TaqMan assays (Applied Biosystems) and Power SYBR Green PCR Master Mix (Applied Biosystems), respectively. Primer details are provided in ESM Table 1. The value obtained for each specific product was normalised to a control gene (cyclophilin A) and expressed as a fold change of the value in control extracts.

Statistical analysis All results are presented as means \pm SEM. Student's unpaired $t$ test was used to compare differences between two groups. Differences between more than two groups were calculated using ANOVA with Fisher's least significant difference (LSD) post hoc test. The researchers were not blinded to experimental groups.

\section{Results}

Differential gene expression changes in macrophages of islets from $d b / d b$ and $o b / o b$ mice The $o b / o b$ mouse maintains normoglycaemia because of successful beta cell compensation, whereas the $d b / d b$ mouse progresses to diabetes due to the failure of beta cell compensation (ESM Fig. 1b). Here, an increase in macrophage levels was observed in islets from diabetic $d b / d b$ mice compared with lean control mice, but not in islets from $o b / o b$ mice (Fig. 1e). This suggests that macrophage accumulation in islets is not solely dependent on obesity or increased fatty acids because these variables are similar in $d b / d b$ and $o b / o b$ mice (ESM Fig. 1a and [4]). We next assessed changes in gene expression in flow-sorted macrophages using RT-PCR. In macrophages from $d b / d b$ islets, the mRNA levels of the proinflammatory macrophage marker interferon regulatory factor 5 (Irf5) were increased, as compared with control (Fig. 1f). In contrast, Irf5 mRNA levels were unchanged in macrophages from $o b / o b$ islets (Fig. 1f). Moreover, the mRNA levels of proinflammatory IL-1 $\beta$
(Illb) were maintained in macrophages from $d b / d b$ islets vs control, whereas they were markedly suppressed in macrophages from $o b / o b$ islets (Fig. 1f). IL-1 receptor antagonist $(I l 1 r n)$ is triggered by the same stimuli as IL-1 $\beta$. Illrn mRNA levels were markedly increased in macrophages from $d b / d b$ islets, whereas they were unchanged in macrophages from $o b / o b$ islets (Fig. 1f). The effects of TGF- $\beta 1$ on macrophages are mainly anti-inflammatory. Interestingly, $T g f b 1$ expression levels were significantly increased in macrophages from $o b / o b$ islets, whereas they were unchanged in macrophages from $d b / d b$ islets (Fig. 1f). The mannose receptor Ctype 1 (MRC1) is expressed on the surface of macrophages and is regarded as a marker of alternative activation. The expression levels of $M r c 1$ were upregulated in macrophages from $d b / d b$ islets, but not in macrophages from $o b / o b$ islets (Fig. 1f). Overall, these findings demonstrate for the first time that markedly different patterns of macrophage gene expression accompany beta cell compensation and failure.

Effects of depleting macrophages on gene expression in islets from $d b / d b$ and $o b / o b$ mice We previously reported that the mRNA levels of genes important for the maintenance of the beta cell phenotype are reduced in $d b / d b$ islets, whereas they are preserved in $o b / o b$ islets [4]. Here, we investigated the effects of depleting macrophages on gene expression in islets from $d b / d b$ and $o b / o b$ mice. The studies were performed ex vivo to assess the direct effects of depleting macrophages in islets. Clodronate-liposome treatment depleted macrophages in islets from control, $d b / d b$ and $o b / o b$ mice (vs PBS-liposome treatment), as evidenced by reduced mRNA expression of macrophage markers, $C d 11 b$ (also known as Itgam) and F4/80 (also known as Adgrel) (ESM Fig. 2a,b and Fig. 2a,b). The mRNA levels of transcription factors important for islet development and the maintenance of beta cell differentiation were increased in clodronate-liposome-treated $d b / d b$ islets compared with PBS-liposome-treated $d b / d b$ islets. $M a f a$ and $P d x 1$ mRNA levels were significantly increased in $d b / d b$ islets after macrophage depletion (Fig. 2c,d). In contrast, no significant difference was observed in the mRNA levels of Mafa and $P d x 1$ in $o b / o b$ islets following depletion of macrophages (Fig. 2c,d). Genes involved in beta cell function were evaluated. The mRNA levels of the glucose transporter GLUT2 (Glut2 [also known as Slc2a2]), the poreforming subunit of the ATP-sensitive $\mathrm{K}^{+}$channel (Kcnj11) and the incretin receptor (Gipr) were increased in macrophage-depleted $d b / d b$ islets compared with PBSliposome-treated $d b / d b$ islets, but they were not significantly affected following macrophage depletion in $o b / o b$ islets (Fig. $2 \mathrm{f}, \mathrm{h}, \mathrm{i})$. These data suggest that macrophage depletion in $\mathrm{db} / \mathrm{db}$ islets is associated with partial restoration of beta cell gene expression. Furthermore, the mRNA levels of adaptive UPR genes, Xbp1, Hspa5, Pdia4 and Fkbp11, were increased in macrophage-depleted $d b / d b$ islets vs $d b / d b$ islets treated with 
PBS-containing liposomes, whereas expression levels of the deleterious UPR effector, Ddit3, were reduced (Fig. 2k-o). In contrast, differences in UPR gene expression were not detected in macrophage-depleted $o b / o b$ islets (Fig. $2 \mathrm{k}-\mathrm{o}$ ). These data indicate that macrophages contribute to the alterations in the pattern of UPR gene expression in $d b / d b$ mice by downregulating Xbp1 and the adaptive UPR, while promoting deleterious UPR gene expression. Moreover, macrophage depletion reduced the expression of genes involved in inflammatory (Illb, Fas and Nfkbia) (ESM Fig. 3) and oxidative stress (catalase [Cat] and heme oxygenase-1 [Hmoxl]) in $d b / d b$ islets (ESM Fig. 4), whereas only catalase was reduced in $o b / o b$ islets. Overall, our studies suggest that depleting macrophages would be beneficial for reducing beta cell stress and preserving beta cell identity.

The therapeutic utility of targeting macrophages for type 2 diabetes is unclear. Recent findings from the Canakinumab Anti-inflammatory Thrombosis Outcome Study (CANTOS) showed that IL- $1 \beta$ inhibition did not have long-lasting effects on $\mathrm{HbA}_{1 \mathrm{c}}$ or fasting plasma glucose levels among patients with diabetes [8]. In our study, insulin secretory function remained impaired in $d b / d b$ islets after macrophage depletion as compared with PBS-liposome treatment (ESM Fig. 5). Another feature of beta cell dysfunction in type 2 diabetes is elevated proinsulin secretion. Interestingly, proinsulin secretion was reduced following macrophage depletion in $d b / d b$ islets as compared with PBS-liposome treatment (ESM Fig. $6 a, b)$. This occurs in concert with restoration of adaptive UPR gene expression (Fig. $2 \mathrm{k}-\mathrm{n}$ ), which is crucial for proinsulin processing [9].

\section{Discussion}

This study shows for the first time that: (1) differential shifts in macrophage genotypes accompany beta cell compensation and failure in obese mice; (2) islet macrophages contribute to beta cell stress and dedifferentiation in a rodent model of diabetes; and (3) islet macrophages are crucial regulators of the balance between adaptive and deleterious UPRs during obesity-associated endoplasmic reticulum (ER) stress. These observations reveal the potential importance of macrophage plasticity in the regulation of beta cell identity during obesity.

Recent studies have shown that the islet-resident macrophage has a homeostatic role [10]. It is not clear whether the increased number of macrophages in $d b / d b$ islets is due to monocyte recruitment. However, if so, one may postulate that infiltrating macrophages may have a less homeostatic function than resident macrophages, which could help explain the major differences in the phenotypes between $d b / d b$ and $o b / o b$ mice. Another possibility is that alternatively activated macrophages could be causing beta cell dedifferentiation in an attempt to regenerate damaged tissue.

Our studies in rodents support a model in which distinct alterations in islet macrophages during obesity are critically important for beta cell abnormalities. We propose that the accumulation of alternatively activated macrophages in islets contributes to beta cell stress and the loss of beta cell identity in diabetes.

Acknowledgements We thank L. M. Thai and E. Lam (Garvan Flow Facility) for technical assistance.

Data availability All data generated and analysed during this study are included in this published article (and its supplementary information files).

Funding This work was supported by a grant from the National Health and Medical Research Council (NHMRC) of Australia and the Diabetes Australia Research Program. JYC is supported by an NHMRC Early Career Fellowship.

Duality of interest The authors declare that there is no duality of interest associated with this manuscript.

Contribution statement JYC and DRL conceived and designed experiments, acquired and analysed data and wrote the manuscript. KL, ELM and CL acquired and analysed data and critically reviewed the manuscript. All authors approved the final version of the manuscript. DRL is the guarantor of this work.

Publisher's note Springer Nature remains neutral with regard to jurisdictional claims in published maps and institutional affiliations.

\section{References}

1. Bensellam M, Jonas JC, Laybutt DR (2018) Mechanisms of betacell dedifferentiation in diabetes: recent findings and future research directions. J Endocrinol 236:R109-R143

2. Talchai C, Xuan S, Lin HV, Sussel L, Accili D (2012) Pancreatic beta cell dedifferentiation as a mechanism of diabetic beta cell failure. Cell 150:1223-1234

3. Engin F, Nguyen T, Yermalovich A, Hotamisligil GS (2014) Aberrant islet unfolded protein response in type 2 diabetes. Sci Rep 4:4054

4. Chan JY, Luzuriaga J, Bensellam M, Biden TJ, Laybutt DR (2013) Failure of the adaptive unfolded protein response in islets of obese mice is linked with abnormalities in beta-cell gene expression and progression to diabetes. Diabetes 62:1557-1568

5. Donath MY, Dalmas E, Sauter NS, Boni-Schnetzler M (2013) Inflammation in obesity and diabetes: islet dysfunction and therapeutic opportunity. Cell Metab 17(6):860-872. https://doi.org/10. 1016/j.cmet.2013.05.001

6. Ehses JA, Perren A, Eppler E et al (2007) Increased number of isletassociated macrophages in type 2 diabetes. Diabetes 56:2356-2370 
7. Eguchi K, Nagai R (2017) Islet inflammation in type 2 diabetes and physiology. J Clin Invest 127:14-23

8. Everett BM, Donath MY, Pradhan AD et al (2018) Antiinflammatory therapy with canakinumab for the prevention and management of diabetes. J Am Coll Cardiol 71:2392-2401

9. Lee AH, Heidtman K, Hotamisligil GS, Glimcher LH (2011) Dual and opposing roles of the unfolded protein response regulated by IRE1alpha and XBP1 in proinsulin processing and insulin secretion. Proc Natl Acad Sci U S A 108:8885-8890
10. Ferris ST, Zakharov PN, Wan X et al (2017) The islet-resident macrophage is in an inflammatory state and senses microbial products in blood. J Exp Med 214:2369-2385 